\title{
Research on Intelligent Power Automation Technology Based on Edge Computing of Power Internet of Things
}

\author{
Su Zhiyong, Lai Weiping, Zhang Yanghua, Huang Yanshan \\ State Grid Info-Telecom Great Power Science and Technology Co.,LTD, Fuzhou Fujian 350003, \\ China
}

\begin{abstract}
With the rapid development of modern economy and the rapid promotion of science and technology, electricity is the realistic need for further transformation of various industries in contemporary society. Because the traditional power management mode is relatively backward and the automation level of different industries is uneven, these will limit the development of intelligent power automation. Therefore, based on the edge computing of power Internet of Things, this paper studies intelligent power automation technology, which is of great value for realizing power automation and improving the management level of power equipment. In this paper, aiming at the research of intelligent power automation technology, based on the edge computing of power Internet of Things, the Internet of Things system based on RFID technology is studied. This system constructs the basic architecture of Internet of Things for smart power applications. The architecture is composed of sensing layer, network layer and application layer, which is used to realize information collection, identification and transmission, and its application in the production management of power Internet of Things. This paper analyzes the functional requirements, factors to be considered, main features and main functions of power equipment management system, gives the design ideas of power equipment management system, and designs an intelligent power equipment system based on edge computing of power Internet of Things. The research results show that RFID technology based on edge computing of power Internet of Things is feasible to realize intelligent power automation, can solve some problems in current power technology, and is of great significance to realize intelligent power automation.
\end{abstract}

Keywords: Power Internet of Things Edge Computing, Intelligent Power Automation, Radio Frequency Identification, Power Equipment

\section{Introduction}

The demand for electricity in various industries continues to increase, the reliability of power supply is gradually improved, and the power grid operation is under great pressure, which undoubtedly tests the security and stability of the power grid system. There are many factors that affect the stability and security of power system, among which the important 
factors include power generation, transmission, substation, distribution, power consumption and rational scheduling of power resources. Equipment and equipment management play a very important role in the production activities of power enterprises. It is related to the improvement of mechanization and automation level of power enterprises, and whether the production, transmission and consumption of electric energy can be carried out continuously and smoothly. Therefore, it is of great value to realize intelligent power automation.

With the development of industry in the world, smart grid research projects have been carried out all over the world. Abroad, Kehoe proposed an LTE memory optimized replication protocol and designed an edge cloud architecture called REPLISOM. By relaxing the LTE environment and efficiently replicating memory objects to edge servers, the delay can be reduced [1]. Tian put forward the development focus and roadmap of smart grid, and advocated that the key development direction of smart grid should be optimizing grid operation mode, improving utilization efficiency of grid infrastructure, large-scale renewable energy grid connection, grid communication information technology, efficient distribution network and fully competitive power market [2]. In China, Xiong Rui has vigorously carried out practical activities of intelligent substations. Greatly improved the overall layout and main connection mode, built an integrated business system and enriched advanced application functions, and the intelligent level of the transformed substation reached the international leading level [3]. Zhao Xiaoyun independently developed and popularized the power grid dispatching system with the widest coverage and the highest degree of automation in the world, which effectively guaranteed the safe and stable operation of the power grid [4].

Based on the edge computing of power Internet of Things, aiming at the research of intelligent power automation technology, this paper systematically studies the Internet of Things system based on radio frequency identification technology. This paper analyzes the development history and current situation of intelligent power automation and Internet of Things at home and abroad, objectively analyzes the current situation of power equipment management system, gives the main ideas of power equipment management system design, and designs an intelligent power equipment system based on edge computing of power Internet of Things. The research results show that the Internet of Things technology based on RFID is feasible and significant to realize intelligent power automation.

\section{Research on Intelligent Power Automation Technology Based on Edge Computing of Power Internet of Things}

\subsection{Advanced Communication Technology}

Data acquisition, protection and control of smart grid are based on high-speed, two-way, real-time and integrated communication system. Communication system and power grid system should all extend to the user side, and the two systems are closely connected to form the basic network of smart grid [5]. Through advanced communication system, smart grid can exchange and circulate real-time and dynamic data. After the completion of the communication system as the foundation, the reliability of power supply and the utilization rate of assets of the power grid will be greatly improved, thus increasing the value of the power grid.

\subsection{Edge Calculation}

Edge computing refers to an open platform that integrates network, computing, storage and 
application core capabilities on the side close to the source of objects or data, and provides services nearby. Its applications are launched on the edge side, resulting in faster network service response and meeting the basic needs of the industry in real-time processing, intelligent application, security and privacy protection [6]. Edge computing is between physical entities and industrial connections, or at the top of physical entities, while cloud computing can still access the historical data of edge computing.

If cloud computing is compared to the brain of the whole computer intelligent system, then edge computing is the eyes, ears, hands and feet of this system. Core server makes intelligent system have strong artificial intelligence, but if this artificial intelligence is deaf and blind, it can't play much role. One of the problems often faced in big data applications is that no suitable data is collected. Edge computing can provide the most accurate and timely data source for the big data algorithm of the core server.

\subsection{Radio Frequency Identification Technology}

Radio frequency identification (RFID) technology is a communication technology that can use wireless signals to identify designated targets and record corresponding data without establishing any connection between the system and the identified objects. When the radio frequency tag passes through the identification area of the reader-writer, it will receive the radio frequency signal sent by the reader-writer, and then the passive radio frequency tag will use the electromagnetic energy in the signal to return one or more EPC codes stored by itself to the reader-writer. Without direct contact, we can easily obtain the item information stored in the radio frequency tag through the reader, and the reader can also write the information into the radio frequency tag [7]. The so-called EPC code refers to the unique code set by the global unified identification system for every single item in the world, which is equivalent to our ID number. The EPC code of each item is unique and contains all aspects of information of the item.

Radio frequency identification has the following characteristics: fast scanning; Miniaturized in size and diversified in shape; Anti-pollution ability and durability; Can be reused; Penetrating and barrier-free reading; The memory capacity of data is large; Security. Radio Frequency Identification (RFID) technology has attracted much attention because of its long-distance reading and high storage capacity[8].It can not only help an enterprise to greatly improve the efficiency of goods and information management, but also interconnect sales enterprises and manufacturing enterprises, so as to receive feedback information more accurately, control demand information and optimize the whole supply chain.

\subsection{Intelligent Control Technology}

Advanced control technology refers to the equipment and calculation method used to monitor, analyze and judge the operation state of power grid in order to prevent power supply interruption and maintain power quality. This technology not only manages the power flow of the whole system, but also provides corresponding control methods for the transmission and distribution side. Advanced control technology can not only improve the management level of assets, but also support the market quotation technology [9]. Advanced control technology is complementary to the other four key technologies. Advanced control technology can monitor most basic components online, diagnose most faults quickly and respond in time.

In the future, advanced control technology will also add preset expert systems to enhance diagnosis and monitoring capabilities. Within the allowable range, the control system will achieve the second-level response speed required by the self-healing power grid, which will undoubtedly greatly improve the reliability of power supply. Advanced control 
technology can not only promote the development of high-speed communication system, but also provide a platform for the development of distributed intelligent analysis software and other intelligent software[10].

\section{Experimental Research on Intelligent Power Automation Based on Edge Computing of Power Internet of Things}

\subsection{Experimental Data Collection}

Network throughput is an important index used and adopted in many power grid experiments, which indicates the total number of data packets sent by a node or unit from the time when sending data information to the time when the information arrives at the destination node. This paper takes the success rate of sending data packets as a measure of power automation level.

The data collected in the experiment are as follows: the success rate of power system with RFID technology and without RFID technology in 50 seconds is $40 \%$ and $20 \%$, respectively; $50 \%$ and $30 \%$ respectively at 100 seconds; $67 \%$ and $46 \%$ respectively at 150 seconds; At 200 seconds, they are; $80 \%$ and $65 \%$ respectively at 250 seconds; At 300 seconds, they were $88 \%$ and $72 \%$, respectively.

\subsection{Experimental Design}

(1) Design of power equipment management system

In view of the problems raised in the previous chapters, when designing the power equipment management system, it should include file management module, defect management module, overhaul management module, etc. Generally speaking, the equipment management system takes equipment file and equipment defect management as the main line, starting from the equipment operation situation reported by the production and operation unit, to the equipment condition inquiry and analysis, etc., for equipment defect inquiry, defect elimination production plan, defect elimination result assessment and overhaul progress. We should also consider such factors as advanced technology, economic rationality and complexity reduction.

(2)Intelligent power equipment system design based on edge computing of power Internet of Things

The equipment management system for smart grid can realize the collection, transmission and operation of label data, and timely transmit the grid operation parameters to the operators, which is convenient for the operators to master and analyze the state of electrical equipment and complete the operation process. The main components of the system include: radio frequency tag, which is mainly composed of large-scale integrated circuit chip with identification code and transceiver antenna; A reader/writer, which can not only read or write data, but also provide necessary power to the radio frequency tag; CPU module, the core of the equipment management system, controls the whole operation of the computer by receiving data, processing data and sending instructions; Communication module to ensure accurate and rapid transmission of information; The alarm module is used for judging and processing unconventional signals and playing a role in protecting the whole system; Server, whose function is to achieve the purpose of data processing and storage by responding to service requests; The monitoring terminal is mainly used for the real-time monitoring service of smart grid equipment management system. 


\section{Experimental Research and Analysis of Intelligent Power Automation Based on Edge Computing of Power Internet of Things}

\subsection{Comparison of Several Near Field Communication Technologies}

Near field communication technology mainly deals with the problem of limited transmission distance, which generally involves wireless communication within tens of meters, and has the characteristics of low cost, low power consumption, wireless communication and peer-to-peer. Mainly used in homes, offices, airports, shopping malls and other places to improve people's living standards and work quality. At present, the short-range wireless communication technologies mainly include ultra-wideband wireless communication technology (UWB), Bluetooth technology, wireless local area network (Wi-Fi), infrared data communication IrDA and other wireless communication technologies, which have developed rapidly and play an irreplaceable role in various fields. The relevant indicators of each wireless communication technology are different, and the applicable scenarios are different. As shown in Table 1.

Table 1. Comparison of Several Short-range Wireless Communication Technologies

\begin{tabular}{|c|c|c|c|c|}
\hline & UWB & Bluetooth & Wi-Fi & IrDA \\
\hline Working frequency & $2.4 \mathrm{GHZ}$ & $2.4 \mathrm{GHZ}$ & $5.2 \mathrm{GHZ}$ & $\begin{array}{c}\text { Wavelength } \\
820 \mathrm{nM}\end{array}$ \\
\hline Voice/Data & Data & Voice/Data & Data & Data \\
\hline $\begin{array}{c}\text { Maximum power } \\
\text { consumption(mW) }\end{array}$ & $1-3$ & $1-100$ & 100 & $1-10$ \\
\hline $\begin{array}{c}\text { Transmission } \\
\text { speed(Mbit/s) }\end{array}$ & 30 & $1 / 2 / 3$ & 55 & $1.54 / 5 / 14$ \\
\hline Transmission mode & $\begin{array}{c}\text { Point to } \\
\text { multipoint }\end{array}$ & $\begin{array}{c}\text { Point to } \\
\text { multipoint }\end{array}$ & $\begin{array}{c}\text { Point to } \\
\text { multipoint }\end{array}$ & Point to point \\
\hline
\end{tabular}

As an advanced wireless carrier communication technology, UWB is characterized by low power consumption. UWB uses nanosecond non-sinusoidal narrow pulses for data transmission, which makes it occupy a wide spectrum range and can transmit signals over a wide bandwidth. UWB is widely used indoors and in dense places. Ultra-wideband technology has the characteristics of large system capacity, strong multipath resolution, high-speed data transmission, accurate positioning, strong anti-interference ability, low power consumption and low cost.

Bluetooth technology works in three frequency bands: industry, science and medical treatment. Its greatest feature is low cost and short distance. Ericsson began to study Bluetooth technology as early as 1994. As a new wireless communication module, Bluetooth technology can connect mobile phones and their accessories and other electronic devices to communicate with each other, and the transmission distance is generally less than $10 \mathrm{~m}$.

As the main technical standard of WLAN at present, the goal of WLAN is to provide access to WLAN, which can complete wireless access from several megabytes to tens of megabytes. The biggest advantage of WLAN is portability. Its coverage is stronger than Bluetooth, up to 100 meters, and it is widely used in office and campus.

IrDA is a point-to-point communication technology using infrared rays. IrDA specifies that the transmission rate of wireless devices has gradually developed from $115.2 \mathrm{kbit} / \mathrm{s}$ to $4 \mathrm{Mbit} / \mathrm{s}$ and $16 \mathrm{Mbit} / \mathrm{s}$.. The receiving angle has been increased from 30 degrees to 120 degrees. Because of the point-to-point connection, there will be little interference when data is transmitted. 


\subsection{Comparison of Network Throughput}

In this paper, the power system using radio frequency identification technology is compared with the common power system, and the success rate of sending data packets between them is obviously different, as shown in Figure 1.

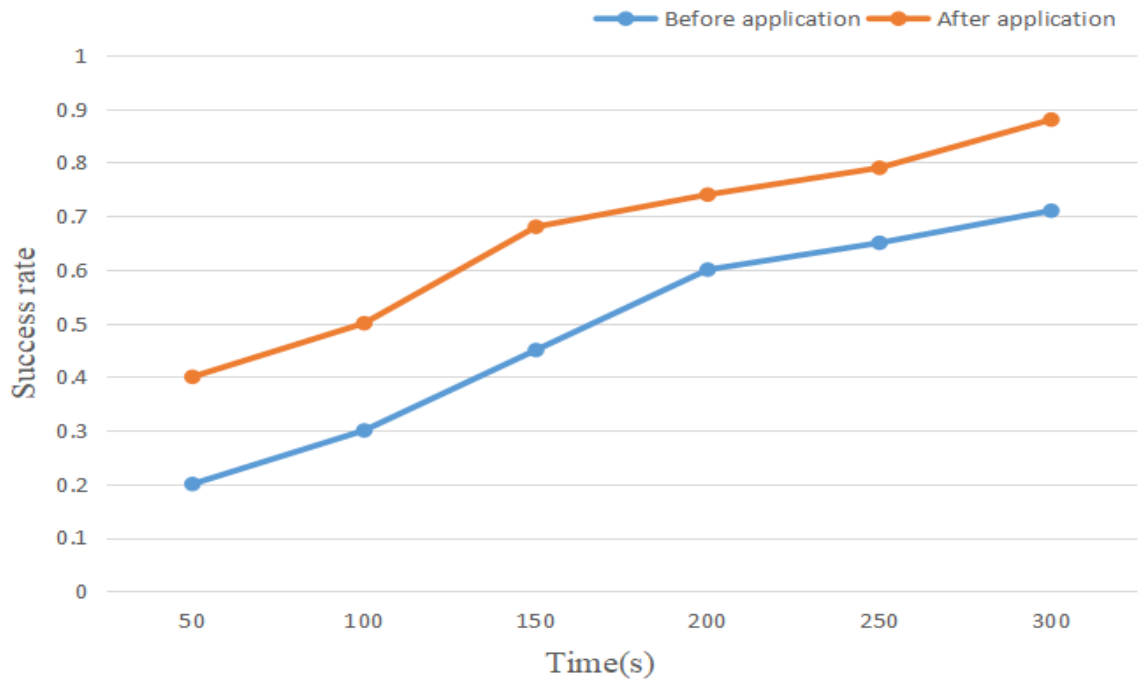

Figure 1. Comparison of network throughput

With the passage of time, the success rate of sending data packets is increasing gradually, but there is a big gap between them. The success rate of power system with RFID technology is much higher than that without RFID technology, so RFID Internet of Things technology can greatly promote the realization of intelligent power automation.

\section{Conclusions}

In this paper, intelligent power automation is studied based on the edge computing of power Internet of Things. Using the Internet of Things technology based on radio frequency technology, the detailed information of the equipment is obtained through radio frequency tag identification, and the flow information is automatically generated and input into the database. At the same time, the relevant technical standards and specifications of radio frequency tag technology and Internet of Things application, as well as information security protection in Internet of Things, are the key factors affecting intelligent power automation. The research content of this paper will promote the development of intelligent power automation technology to a certain extent. This technology needs to be continuously developed, combined with more other research results, and continuously improved and improved.

\section{References}

1. Kehoe, Ben, Patil, Sachin, Abbeel, Pieter, Goldberg, Ken.A Survey of Research on Cloud Robotics and Automation[J].IEEE Transactions on Automation Science and Engineering,2015,12(2):398-409.

2. Tian, J., Gao, M., Ge, G..Research on Intelligent Optimization Control Method for Oil Pumping[J].Advances in Mechanical Engineering,2015,6(0):926958-926958. 
3. Xiong Rui, Sharkh Suleiman, Zhang Xi .Research Progress on Electric and Intelligent Vehicles [J]. Energies,2018,11(7):1762-.

4. Zhao Xiaoyun, Tuo, Xianguo, Ge, Qing, Peng, Ying.Research on the high power cyclotron-wave rectifier[J].Physics of Plasmas,2017,24(7):073117.

5. Li, Xiao Hua, Li, Wen Yi.The Research on Intelligent Monitoring Technology of NC Machining Process[J].Procedia CIRP,2016,56(Complete):556-560.

6. Shanmugham, Shanthi Rekha,Paramasivam, Saravanan.Survey on power analysis attacks and its impact on intelligent sensor networks[J].IET Wireless Sensor Systems, 2018,8(6):295-304.

7. Liu, Derong.The 12th World Congress on Intelligent Control and Automation [Conference Reports][J].IEEE Control Systems,2016,36(6):110-111.

8. Kim, K. J., Lee, Y.-C., Park, J.-H..Fundamental research on power train systems for electric vehicles[J].Materialwissenschaft und Werkstofftechnik, 2015,46(4-5):414-419.

9. Park, Mingon, Hirata, Yoshinori.Research on generation of micro-plasma arc and its power intensity [J].Welding International,2017,31(4):284-290.

10. He Huacan, Zhou Yanquan, Chen Zhicheng. Research on Mathematical Dialectical Logic for Intelligent Information Processing[J].Proceedings,2017,1(3):149. 